\title{
Subjective Causality and Counterfactuals in the Social Sciences: Towards an Ethnographic Causality?
}

\begin{abstract}
The paper explores the role that subjective evidence of causality and associated counterfactuals and counter potentials might play in the social sciences where comparative cases are scarce. This scarcity rules out statistical inference based upon frequencies and usually invites in depth ethnographic studies. Thus, if causality is to be preserved in such situations, a conception of ethnographic causal inference is required. Ethnographic causality inverts the standard statistical concept of causal explanation in observational studies whereby comparison and generalisation, across a sample of cases, are both necessary prerequisites for any causal inference. Ethnographic causality allows, in contrast, for causal explanation prior to any subsequent comparison or generalisation.

Keywords: Causality, Ethnography, Bayesian Narratives, Counterfactuals, Counterpotentials
\end{abstract}

This paper explores the role that subjective statements about causality and their associated subjective counterfactuals and counter-potentials may be allowed to play in the social sciences, where ethnographic techniques and the "social construction" of causality are appropriately invoked. The background to this exploration is the theory of Bayesian Narratives (Abell, 1987; 2009a; 2009b) where subjective statements may be used as evidential items (amongst others) in Bayesian causal inference. Such inferences are required when frequency based statistical approaches are impossible because of limited numbers of comparative cases (units of analysis) and a singular ethnographic concept of causality must inevitably be deployed in each case. Any subsequent limited generalisation, usually across a small number of cases, will place causal inference (explanation) as logically prior to both comparison and generalisation. Ethnographic researchers usually suggest that “justified belief", rather than "objective truth" (Cardano, 2009), is generated in social interactions 
between the observed informant and the ethnographer. ${ }^{1}$ The inquiry will be pursued with this in mind.

The concept of causality is, of course, itself controversial amongst ethnographers, many of whom seek to disavow the concept altogether, remaining content with "an understanding of the meaning of human actions" which is largely conceived as a descriptive exercise which rejects "why questions" (Small, 2013). Nevertheless, Abend et al. (2013) find that many ethnographic studies do entertain some concept of causality though the precise method of establishing a causal inference from ethnographic data remains rather difficult to fathom. In addition the extensive literature on qualitative, small- $N$ case based research has engaged with concepts of causality but almost invariably in a comparative perspective where $N>1$ and where the language of variables (if only nominal dichotomies) is resorted to (Mahoney, 2000; 2012; Mahoney et al 2013; Ragin,1987). In this paper we concentrate upon situations which ethnographers might wish to describe as unique and where the logic of comparison across cases is initially absent. If a concept of causality can be found which is reasonably faithful to the precepts of ethnography, then surely it should be explored to enable explanatory studies (Elman et al, 2016; Rohlfing, 2013). Such enablement will inevitably require us to discard the standard explanatory procedure whereby comparison across cases is a necessary prerequisite for any causal explanation. Indeed, in so far as comparison may be involved, the approach developed in this paper places it as posterior to the prior establishment of a causal explanation in each comparator (Abell, 2009a). We have adopted the term Ethnographic Causality in the full recognition that some would want to name the procedure alternatively.

Here we adopt what may be called a "mechanism approach" to causality whereby human activity provides the causal connection (motor energy) between the causal and outcome/effect states (Goldthorpe, 2001; Hedström, 2005). We, thus, examine causal links (designated as an arrow: $\rightarrow$ )

\footnotetext{
${ }^{1}$ The authors are far from conversant with all the details of ethnographic methods but the paper is written in a manner that tries to take seriously some of the critical claims that ethnographers and others make about frequency based statistical methods in the social sciences whilst maintaining the aspiration to reap the clarity benefits of formal explication
} 
which take the general form $X_{c} \rightarrow \cdot D X_{o} \rightarrow Y$ where both $X_{c}$ and $Y$ may comprise sets of conjunctions of events, $\cdot$ stands for a specified actor (individual/collective) and $D X_{o}$ describes an action or forbearance designed to bring about $Y .{ }^{2}$ Causal links of this sort may then be strung together in narratives. It should be clear that, despite the similarity in expression, the term $\cdot D X_{o}$ is not an application of Pearl's (2009) hugely influential, do-calculus whereby the causes of $D X_{o}$ would be held constant in the DAG structure.

Nevertheless, there is an immediate parallel between the formulation $X_{c} \rightarrow \cdot D X_{o} \rightarrow Y$ and the treatment of intervening mediator variables $X \rightarrow M \rightarrow Y$ in the Large- $N$ tradition. Furthermore, using Pearl's (2009) backdoor criterion, independent mediator variables have the marked virtue that, whilst they can protect the causal interpretation of the co-variation between $X$ and $Y$ from ubiquitous unmeasured confounders of $X$ and $Y$, though they cannot so protect against confounders of $X$ and $M$, and $M$ and $Y$ (Rubin, 2005). It might prove constructive to see how ethnographic causality might contribute to causal inference when this is the case. ${ }^{3}$

The extensive debates in various literatures about the nature of causal inference has often been limited to the context of a Large- $N$ frequency framework. Breaking with this tradition in the direction of what we may cautiously call singular causality is full of hazards. This may account for the fact that a small- $N$ concept of causality, consistent with "uniqueness" $N=1$, has not been forthcoming in the literature. ${ }^{4}$ Furthermore, this has stymied the interplay of small and Large$N$ studies where case studies are designed to act as a hand maiden to frequency based studies.

The paper will proceed as follows. Firstly, the nature of subjective causal, counterfactual and counter potential statements is briefly reviewed. Secondly, a small illustrative (and only illustrative)

\footnotetext{
${ }^{2}$ Some readers may prefer the formulation where actions are named in terms of their objectives. The connection between actions and their objectives has attracted much attention from philosophers in terms of the logical connection argument, namely that the connection is analytic and not contingent and therefore not causal. However, we shall interpret it as causal as nothing is lost by doing so. The following analysis could be pursued by substituting $Y$ with $X_{o}$.

${ }^{3}$ The possible complementary roles of ethnographic (case study) causality and frequency based causality will be taken up in a subsequent paper.

4 Attempts to formalize singular causality have been conducted by Hitchcock (2007) and Cartwright (2017), among others.
} 
empirical study is introduced. Thirdly, Bayesian Narratives are briefly outlined. Fourthly, the process of Bayesian inference to credible beliefs is examined. Fifthly, the inference through credible beliefs to causal connections is outlined. Sixthly, the conception of meta-ethnography is introduced. Then the illustrative empirical study is reintroduced and finally the paper briefly concludes.

\section{Subjective Causal, Counterfactual and Counter Potential Statements}

Assume an observer/ethnographer elicits statements from an actor about her/his completed action, namely doing $X_{o}$, as follows:

(1) "I $\operatorname{did} X_{O}$ because of $X_{C}$ to realise $Y$ " (a subjective first person singular causally related statement);

(2) "I would not have done $X_{o}$ (i.e. forborne to do $X_{o}$ ) to realise $Y$, if $X_{c}$ had not happened" (a singular first person subjective counterfactual). ${ }^{5}$

Alternatively, the ethnographer might elicit information, from the actor about his/her future anticipated course of action as follows:

(3) "If $X_{c}$ happens then I will do $X_{\mathrm{o}}$ to realise $Y$ ",

(4) "If $X_{C}$ does not happen then I will not do (forbear to do) $X_{o}$ to realise $Y$ ".

Both $X_{c}$ and $Y$ may comprise of more or less complex descriptions, which are proffered in the actor's own descriptive language (discourse) which we may assume will be derived from his /her own cultural heritage. ${ }^{6}$ The statements (1) to (4) may also reflect the actor's uncertainty and

\footnotetext{
${ }^{5}$ Simple statements like these brush aside some of the complexities that might arise because "doing $X_{o}$ " covers both motivational/intentional and beliefs about objectives $(Y)$, sometimes modelled as the practical syllogism (Von Wright, 2004; Abell, 1987). Thus, the reasoning takes the form: the actor, in situation $X_{c}$, intended $Y$ and the actor believed that doing $X_{o}$, in $X_{c}$, would result in (cause) $Y$. Furthermore, it may be important to distinguish forbearing to do something and not doing something. The former will be used to imply intention and, thus, conscious deliberation not to do, the latter not so. Clearly we do not do all those things we could do on an occasion but probably only consciously think of a few of these. Note also since Error! Main Document Only.Error! Main Document Only.Error! Main Document Only. $X_{c}$ and Error! Main Document Only.Error! Main Document Only.Error! Main Document Only. $Y$ both stand for sets of conjoined events, the negation of a set in a counterfactual statement implies that if any one of the conjoined elements in the set is absent then the causal connection will be absent. $X_{c}$ may perhaps be conceived as comprising of INUS causal conditions (Mackie, 1980)

${ }^{6}$ Translating between the informants' and ethnographers' vocabularies raises issues ignored in this paper. In a single case there is perhaps no good reason for the ethnographer to impose terms, unlike in generalising studies. Casual empiricism suggests that objectives $(Y)$ are easier to solicit than causal conditions $\left(X_{c}\right)$. Prompting is often necessary to solicit the latter. The statements can also be expressed in differing ways whilst still conveying essentially the same information. We shall assume the alternative possible locutions (i.e. framing) are logically equivalent for the purposes of causal inference unless otherwise stated. Furthermore, $X_{c}$ may be given as the absence of $Y$. This allows that the absence of states can be the causal prompt (Ragin, 1987). It should be emphasised that the use of symbols here $\left(X_{c}\right.$ and $Y$ ), should not be taken to imply that the analysis can be carried out in terms of "variables", the symbols are characteristically short-hand for
} 
consequently be expressed in a probabilistic form. Furthermore, statements may also derive, not from the actor commissioning the action, but from alternative informants claiming information about the focal action, which will generate subjective statements along the lines of "because of $X_{c}$ he/she did $X_{o}$ to realise $Y$ ", "because of $X_{c}$ we $\operatorname{did} X_{o}$ to realise $Y$ " and " because of $X_{c}$ they did $X_{o}$ to realise $Y$ ". In addition, corresponding counterfactual versions of these statements may also be elicited. All these various statements may also be tensed.

The key logical point is that subjective elicitation apparently surrenders information about both the cause and counterfactual for the same unit of analysis, namely the actor commissioning the action. In this respect, if credibility can be ascertained, there is a clear advantage of such data over much comparative statistical data in many observational studies where intra-unit comparisons are not possible; though Pearl's (2009) do-calculus does provide a route to intra-unit counterfactuals.

The problem we face is: under what assumptions may an analyst, who might or might not be the ethnographer, allow elicited subjective statements to stand as credible evidence for a justified retro-predictive belief that:

(5) $X_{C}$ caused the actor to do $X_{o}\left(\cdot D X_{o}\right)$ which caused $Y$.

Or prediction that:

(6) $X_{C}$ will cause the actor to do $X_{o}\left(\cdot D X_{o}\right)$ which will cause $Y$.

It is important to note that, from an ethnographic standpoint, the subjective evidence, namely the causal and counterfactual statements themselves, must explicitly be associated with a specified ethnographer. Ethnographic principles require an acknowledgment that the informant's statements are generated by virtue of the social interaction of the informant and ethnographer wherein the

complex natural language descriptions. Furthermore, again casual empiricism has revealed that actors may offer alternative causal mechanisms like: "I would also have done $X_{o}$ if $Z$ " and "I would not have done $X_{o}$ if neither $X_{c}$ nor $Z$ ". It is imperative to observe that because Narratives (see below) are depicted as "and" not as "or" directed a-cyclic graphs, alternative causes are not covered. This is appropriate because in a single case alternative causal mechanisms are not logically possible. Although this would, in the recent past, have drawn a sharp line between the old regression based additive structural modelling and interaction structures, with the movement to non-parametric modelling of DAGs this distinction falls away (Pearl, 2000; Morgan and Winship, 2015). 
credibility of the informant and the informant's statements come to be assessed by the ethnographer. Clearly how and why ethnographers assign credibility is a complex issue which warrants further attention.

Several points of initial clarification should be made. Firstly, some ethnographers would immediately cavil at the inferences to causality between set $X_{C}$ and $\cdot D X_{o}$ suggesting that voluntary actions and causality are logically incompatible. However, we may assume that the above subjective statements can, in principle, be supplemented by subjective counter-potential statements which run as follows:

(7) "I (she/he, we, etc.) could have not done $X_{o}$ (forborne to do $X_{o}$ ) if $X_{c}$ ",

(8) "I (she/he, we, etc.) could have done $X_{o}$ if not $X_{c}$ ".

Similarly, the future tense statements can also be accompanied by counter-potentials. We may assume that counter-potentials preserve the voluntary nature of human action whilst maintaining the possibility of an inference to causality. That is to say, though informants can speak of why actors did /will do $X_{o}$ (forbear to do $X_{o}$ ), they fully recognise that the actor could always have done (may do) otherwise.

The attraction of the various subjective statements outlined above is that they can all apparently be acquired by an ethnographer relating to a specific action (forbearance). Thus, if they are mutually understood by an ethnographer and an informant and deemed as credible by the former, they open a route (albeit only probabilistic) to causal inference without the need to generalise across comparative units. That is to say, as we have noted above, causality can then, in principle, be justified in the absence of comparators and statistical co-variation. This allows that single case studies may, if handled carefully, surrender causal information and causality (explanation) may then be generalised by comparing a number of cases. The procedure is only appropriate when the number of cases falls short of a statistical sample. Everything depends, however, upon the credibility afforded to the subjective statements. How should they be elicited and then treated as credible evidence for a justified causal inference? 
Under what conditions may we assume the informant understands what the causes and objectives of his own and others actions are and is able to impart this understanding to the ethnographer in virtue of the elicitation of subjective statements? Certainly, if the vocabulary in which the elements of sets $X_{c}$ and $Y$ are expressed is that of the informant then this is more likely to be the case and ethnographic principle enjoins precisely this as the starting point for any research. Scepticism always remains, however, as to whether social scientists can assume a causal understanding, amongst informants, of their own and others' actions. This scepticism may be particularly acute in respect of the counterfactuals and counter-potentials. Do people know what they and others would have done in the absence of $X_{c}$ and are they capable of conveying this information to the ethnographer?

If we switch to prediction rather than retro-diction then things are not quite as bleak because the ethnographer can treat subjective statements as predictive and test this assumption if and when appropriate circumstances arise. Nevertheless, the conditions under which subjective causal, counterfactual and counter-potential statements can be relied upon as sources of credible evidence are far from transparent. Furthermore, when multiple ethnographers are introduced alongside multiple informants into the mix then the problems of comparing the likely varying elicited statements, with a view to compendious causal inference, clearly exacerbates the inferential problems. We hope to take these issues up in a subsequent paper.

\section{An Illustrative Empirical Example: An Initial Look}

We introduce here an illustrative empirical example which will be explored in more detail later in the paper. ${ }^{7}$ In a study of producer cooperatives in developing countries explanations were sought as to why many failed whereas very few prospered (Abell, 1990). Attempts to find a statistical model to account for this asymmetric distribution, which was generalizable across cases proved elusive. To

\footnotetext{
${ }^{7}$ It must be emphasised that the example is only a simplified illustrative model introduced to facilitate an understanding of the analysis in this paper. It is not proffered as a definitive study and should not be interpreted as such.
} 
put it succinctly, each case appeared to be rather historically unique and a subsequent in depth study of a single highly successful cooperative lead to the theory of Bayesian Narratives (below). Here we concentrate upon a single action whereby the collective governing board appointed an external professional manager. A senior member of the governing body was asked the question, after a great deal of exploratory discussion, "why was an independent manager appointed". ${ }^{8}$ The answer (whilst improving the expressed English) was as follows:

"Because sales were dropping, the quality of the products was not competitive, and the problems of discipline were uncontrolled, a manager was appointed to improve the allround performance whilst making the cooperative an attractive place to work."

It is important to recognise that this statement was mutually constructed in the interaction of the ethnographer/author and the informant and was endorsed by the informant as a correct and an acceptable causal explanation of the action taken and its outcome. The author/ethnographer was now faced with (1) assessing the credibility of this statement and (2) inferring causality.

Thus, the possible causal inference takes the form:

$$
\begin{aligned}
& \text { Set } X_{c}=\left\{X_{c 1}, X_{c 2}, X_{c 3}\right\} \\
& X_{c 1}=\text { sales dropping, } \\
& X_{c 2}=\text { uncompetitive quality of products, } \\
& X_{c 3}=\text { discipline problems. } \\
& D X_{o}=\text { appoint an independent manager. } \\
& \text { Set } Y=\left\{Y_{1}, Y_{2}\right\} \\
& Y_{1}=\text { improve all round performance, } \\
& Y_{2}=\text { attractive place to work. }
\end{aligned}
$$

$\left\{X_{c 1}, X_{c 2}, X_{c 3}\right\} \rightarrow$ Governing Body $D X_{o} \rightarrow\left\{Y_{1}, Y_{2}\right\}$

\footnotetext{
${ }^{8}$ Cooperative principles normally require that managers are appointed from within the membership. Thus, recruiting an independent manager very much went against established procedures.
} 
Where the author of $D X_{o}$ is a collective actor, namely, the governing body, which appointed a manager to improve performance and the attractiveness of the workplace. When faced with this inferred causality an informant member of the governing body (with prompting) stated;

"If falling sales, uncompetitive products and discipline problems had not been the case then we (the governing body) would not have appointed an independent manager";

Namely, he proffered a subjective counterfactual. Data on counter potentials was unfortunately not gathered. Additional informant statements will be introduced later in the paper.

\section{Bayesian Narratives}

A Narrative (Abell, 1987; 2009b) comprises a time distributed a-cyclic directed "and-digraph" (andDAG) constructed from multiple causal connections of the form $X_{C} \rightarrow \cdot D X_{o} \rightarrow Y$ where the components of set $X_{C}$ comprise the in-degree nodes running into the $D X_{o}$ node and the $Y$ nodes its out-degree. Bayesian Narratives are generated by providing subjective evidence of the sort outlined above, for each of the complex, action generated, causal links in the Narrative. Many of the causal links may involve placing prior actions into set $X_{c}$ and posterior actions into set $Y$, thus capturing the notion of social interaction. $X_{C}$ and $Y$ may then be connected by a single or multiple directed paths of actions thus generating $X_{c} \rightarrow$ Narrative $\rightarrow Y$.

First, note that subjective statements examined above refer to both the complex conjunctions of events $X_{c}$ and $Y$ and their causally generative connecting mechanism - they come as of a piece. However, the evidence will usually bear only an uncertain probabilistic relationship with the existence of the generative causal mechanism. All will depend upon the credibility of the subjective statements, afforded by informants, in the ethnographers' estimations. ${ }^{9}$

Subjective evidence will be available for each action under investigation. The assembly of sets $X_{c}$ and $Y$, constructed from the subjective causal statements, to a degree contrasts with

\footnotetext{
${ }^{9}$ Ethnographers have developed various methods to ascribe "truth values" to assertions provided by informants (Cardano 2009). They have also discussed how to adjudicate between alternative interpretations when confronted with conflicting evidence (Chandra, 2014; Heider, 1988). In what follows we suggest a formalized approach to these issues (compare with Heider, 1988).
} 
procedures in most case studies (and process tracing; Mahoney, 2012) which are usually constructed by initially assembling a chronology of events and subsequently searching for grounds to insert causal connections. The events are accordingly often assembled without initial recourse to an elucidation of actions/interactions which generate them. The procedure advocated here, however, affords primacy to the "understanding of human actions", in the sense of what occasions them and what their objectives are. The understanding of human actions is, of course, an objective promoted by most ethnographers.

The important point to note is that from an ethnographic standpoint the initial causal/ counterfactual subjective statements are elicited as evidential material during the social interaction of the informants and the identified ethnographers. That is to say, the inferences are socially constructed creating posterior beliefs on the ethnographer's behalf, given the perceived credibility of the evidential statements. The degree of credibility, once established by an ethnographer, will constitute a prior for an inference to the hypothesis that there is justified belief in the generative causal link between sets $X_{c}$ and $Y$.

Ethnographic practitioners are assiduous in recording (producing text) charting the details of such interactions and inferences. They would no doubt label this record, if pursued with due diligence, as charting the social construction of the belief (or disbelief) in the credibility of the informants statement and then to justified belief in the causal link.

We need eventually to place this procedure within the framework of Bayesian inference. First, however, the causal states which are members of sets $X_{c}$ and $Y$ must be extracted from the subjective causal statements provided by a number of informants, and perhaps also more than one ethnographer. However, in this introductory paper we will concentrate upon a single ethnographer with multiple informants though the penultimate section will briefly introduce meta-ethnography.

The members of the causal set, $X_{c}$ and outcome set $Y$ may, however, both vary across informants. Then to what extent is there agreement amongst the informants about the states/nodes in, 
Ethnographic Causality

respectively, both sets $X_{c}$ and $Y$ ? If agreement or near agreement across informants fails, then how should the ethnographers proceed? One possibility is to treat each informants' sets as an independent estimates of $X_{C} \rightarrow \cdot D X_{o} \rightarrow Y$. Alternatively, a threshold may be set of the number of informants' endorsements required for a cause to be included in set $X_{c}$ and likewise an outcome in set $Y$ (see below on meta-ethnography). 
The analysis, thus, enables answers to be given, in a systematic manner, as to whether a

particular causal or outcome state is sufficiently endorsed by the informants to be included in

respectively sets $X_{c}$ and $Y$ which are to be subjected to the Bayesian inference. ${ }^{10}$

\section{Bayesian Inference to Credible Beliefs}

Consider the causal linkage between sets $X_{c}$ and $Y: X_{C} \rightarrow \cdot D X_{o} \rightarrow$ Y. Let $s_{i}$ stand for the conjunction

of subjective causal and counterfactual statements elicited from an informant/actori, by

ethnographer $e$. Then let:

$B_{e}=e$ 's prior belief in the credibility of subjective statements elicited from informants, $\neg B_{e}=e$ 's prior disbelief in the credibility of subjective statements elicited from informants. ${ }^{11}$

Given $s_{i}$, then by Bayes' rule. ${ }^{12}$

\footnotetext{
${ }^{10}$ Ethnographers will at this stage in the argument no doubt feel uneasy about the move from natural language statements (discourse) to probabilities. Clearly, we need to ask: what is the epistemic role of probability statements? The paramount reason for moving from discourse to probabilities is that it affords the ethnographer a scaled mechanism for combining various items of evidence (see below). Thus, rather than having a list of similar statements of varying credibility about the causal connection under investigation an aggregate estimate of their causal probity can be constructed. Nevertheless even if the move to probabilities is intellectually sanctioned there still remains a further puzzle as to their epistemic status. Clearly, they cannot usually, but may be, derived from frequency considerations on the ethnographer's behalf. Should we assume that the ethnographer holds probabilities in mind? Perhaps this is reasonable if she/he is trained to think in such a manner? Alternatively, probabilities may be interpreted as an "as if" device enjoined in the practice of social science. The problem with the latter is that "as if" assumptions are usually deemed appropriate when they facilitate generalisation across cases but such is not the prime objective of singular causality. (Generalisation is, of course, involved across evidential items). Although most ethnographers will resist any role for rational choice in the social sciences it is worth noting that the formulation of Bayesian inference based upon subjective probabilities can be given a rational choice interpretation whereby it is deemed that utility maximising individuals should follow the reasoning of Bayesian inference (Howson and Urbach, 2006). Indeed, the issue is somewhat deeper than mere recommendation by virtue of the theorem which shows that preferences with standard properties can always be associated with a probability distribution (Maher, 1993). Bayesian nets have been interpreted as a foundational model for the "epistemic" interpretation of causal inference (Williamson, 2005). However, Bayesian narratives are quite distinct, though consistent with Bayesian nets. The latter usually, though not invariably, depend upon frequency measures and characteristically do not derive the actual causal links from subjective evidence but rather from conditional probabilities between states $\left(X_{c}\right.$ and Error! Main Document Only.Error! Main Document Only.Error! Main Document Only. $Y$ ). Such conditional probabilities can, of course, be derived from narrative connections but the causal conclusions are not derived in this manner.

${ }^{11}$ There may be some contention about how to treat the hypothesis about a generative mechanism which connects $X_{c}$; $D X_{o}$ and $Y$. The subjective statements provide evidence for all three components and their possible causal connections. It would be possible to think independently about the credibility that any subjective statements provide for each causal connection separately. The analysis will, however, be pursued in terms of sets $X_{c}$ and $Y$ connected by $D X_{o}$ in the spirit of the idea of a total generative causal mechanism.

${ }^{12}$ We concentrate upon the odds ratio rather than, for instance, the difference in posterior and prior odds $P\left(B_{e} \mid\right.$ evidence $)-P\left(B_{e}\right)$ or the ratio $P\left(B_{e} \mid\right.$ evidence $) / P\left(B_{e}\right)$, which might seem more intuitive measures. However, with these measures, the incremental impact of an item of evidence when the prior is near its maximum of unity will be less than with the same item when the probability is lower. The odds ratio allows the impact scale to vary from minus to plus infinity which obviates this problem (Schum, 1994).
} 


$$
\begin{gathered}
\frac{P\left(B_{e} \mid s_{i}\right)}{P\left(\neg B_{e} \mid s_{i}\right)}=\frac{P\left(B_{e}\right)}{P\left(\neg B_{e}\right)} \cdot \frac{P\left(s_{i} \mid B_{e}\right)}{P\left(s_{i} \mid \neg B_{e}\right)} \\
\operatorname{Odds}\left(B_{e}: \neg B_{e} \mid s_{i}\right)=\operatorname{Odds}\left(B_{e}: \neg B_{e}\right) \cdot L_{s_{i}} \\
\log \left(\operatorname{Odds}\left(B_{e}: \neg B_{e} \mid s_{i}\right)\right)=\log \left(\operatorname{Odds}\left(B_{e}: \neg B_{e}\right)\right)+\log \left(L_{s_{i}}\right)
\end{gathered}
$$

Where $L_{s_{i}}$ is the likelihood ratio (estimated by $e$ ) of the evidence $s_{i}$ given $B_{e}$ and $\neg B_{e}$. That is;

$$
L_{s_{i}}=\frac{P\left(s_{i} \mid B_{e}\right)}{P\left(s_{i} \mid \neg B_{e}\right)}
$$

Thus, from $e$ 's prior odds and estimation of the likelihood ratio $L_{s_{i}}$, his/her posterior odds in the belief about credibility of $i$ 's statement can be derived on a log interval scale (Abell, 2009b; Schum,1994). Note that whatever the prior beliefs happen to be, if $L_{s_{i}}$ is greater than one then the posterior beliefs are strengthened and vice versa.

If, however, we set the prior odds at unity, which some ethnographers appear, by implication so to do, then the likelihood ratio is numerically equal to the posterior odds. ${ }^{13}$ In this situation $e$ has merely to estimate her/his likelihood ratio. Estimation of this nature might still appear rather demanding of the ethnographer. If, however, the estimate is reported alongside an explicit statement of $s_{i}$ itself, nothing is lost and something may be gained.

Ethnographers, at this juncture, might still cavil - wherein lies the benefit in making this probabilistic estimate, rather than merely reporting $s_{i}$ which would be the standard practice? The answer is that there may be ethnographers, other than $e$, eliciting statements from $i$ and then the question arises as to how their, likely differing beliefs, in the credibility of $i$ 's statements should be

\footnotetext{
${ }^{13}$ Setting the prior odds at unity may appear inappropriate to many Bayesians and it could be dropped in all that follows. However, there is perhaps some justification to be found in ethnographic precepts for so doing. Ethnographic researchers are often enjoined to engage in research without bringing any preconceived ideas to the research site. Whether this is possible is, of course, very much a moot point; but it does apparently invite the suppression of any prior odds one way or the other that $e$ might bring to the analysis. The precept does, we think, flow from the assumption that much social phenomena is ultimately unique and therefore to bring preconceived ideas to the research may distort a full understanding of "what is going on" from the informant's standpoint.
} 
combined? ${ }^{14}$ If in addition there are also multiple informants then how should each of their statements be combined? It is clear that a systematic procedure is required that enables combinations across ethnographers and informants. Placing things on probabilistic scale helps considerably in this respect. The procedure can be reported in a transparent manner such that any audience for the research can appreciate how the conclusion was drawn, which is currently scarcely the case in many ethnographic studies (Abend, et al., 2013).

Let us stay with a single ethnographer $e$, but now introduce $m$ conjunctive, subjective statements $s_{i 1}, s_{i 2}, \ldots, s_{i m}$ deriving from $m$ informants observing/witnessing $\cdot D X_{o}$. Initially, assume that each informant provides the subjective statements independently of each other. So, $e$ can assume that the statements are independently elicited conditional on $B_{e}$ and $\neg B_{e}$. Then:

$$
\begin{gathered}
P\left(B_{e} \mid s_{i 1}, \ldots, s_{i m}\right) P\left(s_{i 1}\right) \ldots P\left(s_{i m}\right)=P\left(B_{e}\right) P\left(s_{i 1} \mid B_{e}\right) \ldots P\left(s_{i m} \mid B_{e}\right) \\
P\left(\neg B_{e} \mid s_{i 1}, \ldots, s_{i m}\right) P\left(s_{i 1}\right) \ldots P\left(s_{i m}\right)=P\left(\neg B_{e}\right) P\left(s_{i 1} \mid \neg B_{e}\right) \ldots P\left(s_{i m} \mid \neg B_{e}\right) .
\end{gathered}
$$

The log odds of $e_{i}$ 's beliefs about the credibility of informants' $m$ statements will take the form:

$$
\log \left(\operatorname{odds}\left(B_{e}: \neg B_{e} \mid s_{i 1}, \ldots, s_{i m}\right)\right)=\log \left(\operatorname{odds}\left(B_{e}: \neg B_{e}\right)\right)+\log (L S),
$$

where $\log (L S)=\sum_{i=1}^{m} \log \left(L_{s_{i}}\right)$. Once again, adopting the ethnographic precept, we may cautiously assume that the prior odds can be set at unity. If this is feasible the posterior odds are then equal to the likelihood ratio $(L S)$ and $e$ can estimate the posterior odds directly rather than inferring such from the prior odds and the likelihood ratio. Ethnographers often assemble evidence in a sequential manner drawing a line at the point when new evidence does not alter conclusions to be drawn.

Dropping the assumption that the evidential statements are independent, conditional on $B_{e}$ and $\neg B_{e}$, does not materially alter the situation except that $L S$ must nowacknowledge the pattern of dependence amongst the subjective statements (Abell, 2009b). Such dependencies are, of course, to

\footnotetext{
${ }^{14}$ Heider (1988) discusses such disagreements between ethnographers and the methodological puzzles that such disagreements pose.
} 
be expected when the evidential statements are obtained for a particular action from multiple informants all involved in the same narrative.

Clearly, it would prove difficult for the ethnographer to estimate each of a string of $m$ likelihood ratios, be they independent or not. Thus, a direct estimate of $L S$, rather than its component likelihood ratios, is perhaps all that can be demanded. ${ }^{15}$ Be this as it may, once again why should the ethnographer go through this exercise at all? The answer is twofold First, as we have observed the aggregation of evidential items is made explicit but, second, differing estimates can be aggregated across ethnographers. So, alternative ethnographers are each endowed with a disciplined framework within which to debate their differing credibility assessments of informants' statements.

\section{From Subjective Statements through Credible Beliefs to Justified Belief in Causal Connections}

If we now allow an inference, by a given ethnographer, from the subjectively provided evidence to a "justified belief" in the probability of the actual existence of the causally generated mechanism $\left(X_{c} \rightarrow \cdot D X_{o} \rightarrow Y\right)$ and label this as hypothesis $H$ and its absence as $\neg H$.We are now interested in:

$$
\operatorname{odds}\left(H: \neg H \mid s_{1}, s_{2}, \ldots, s_{m}\right)=\operatorname{odds}(H: \neg H) \cdot L
$$

where $L=\frac{P\left(s_{1}, S_{2}, \ldots, S_{m} \mid H\right)}{P\left(s_{1}, s_{2}, \ldots, s_{m} \mid \neg H\right)}$.

Thus, as per the above, the ethnographer estimates the likelihood ratio at the aggregate level across all evidential statements. It important, however, to understand the logical structure of such an inference, particularly how it embodies the ethnographer's credible beliefs which do not appear in the equation. We are interested in how credible beliefs in the available evidence do or do not licence inference to the odds $\left(H: \neg H \mid s_{1}, s_{2}, \ldots, s_{m}\right)$.

\footnotetext{
15 The nature of this procedure is, from an inferential standpoint, rather important to record because, if relaxed, the inferences would run from each item of evidence to individual beliefs about the credibility of each informant's statement. In that case any across informant statements about causality would arise in the inference from these beliefs to the existence of a causal link (see below). It is straightforward to adjust the inferential models to credible beliefs in this manner.
} 
It is convenient, however, given our above analysis to consider a single item of evidence $s$, rather than $m$ items and to drop the designation of the ethnographer, $e$, thus reducing the complexity of the notation. We need to examine $L$ in this situation (which is, of course distinct from $L S$ defined above). With this objective in mind:

$$
\begin{gathered}
P(s \mid H)=P(s, H) / P(H) \\
P(s \mid \neg H)=P(s, \neg H) / P(\neg H) \\
P(s, H)=P(s, B, H)+P(s, \neg B, H) \\
P(s, \neg H)=P(s, B, \neg H)+P(s, \neg B, \neg H) \\
P(s, B, H)=P(H) P(B \mid H) P(s \mid B, H) \\
P(s, \neg B, H)=P(H) P(\neg B \mid H) P(s \mid \neg B, H)
\end{gathered}
$$

With similar expressions for $P(s, B, \neg H)$ and $P(s, \neg B, \neg H)$. Thus, substituting (10) in (9),

$$
\begin{gathered}
P(s, H)=P(H) P(B \mid H) P(s \mid B, H)+P(H) P(\neg B \mid H) P(s \mid \neg B, H) \\
P(s, \neg H)=P(\neg H) P(B \mid \neg H) P(s \mid B, \neg H)+P(\neg H) P(\neg B \mid \neg H) P(s \mid \neg B, \neg H)
\end{gathered}
$$

And substituting (11) in (8) we get

$$
\begin{gathered}
P(s \mid H)=P(B \mid H) P(s \mid B, H)+P(\neg B \mid H) P(s \mid \neg B, H) \\
P(s \mid \neg H)=P(B \mid \neg H) P(s \mid B, \neg H)+P(\neg B \mid \neg H) P(s \mid \neg B, \neg H)
\end{gathered}
$$

Thus, our target,

$$
L=\frac{P(s \mid H)}{P(s \mid \neg H)}=\frac{P(B \mid H) P(s \mid B, H)+P(\neg B \mid H) P(s \mid \neg B, H)}{P(B \mid \neg H) P(s \mid B, \neg H)+P(\neg B \mid \neg H) P(s \mid \neg B, \neg H)}
$$

The aggregate estimate by the ethnographer of the ratio $L$ is, thus, logically constituted from constituent likelihood ratios. In a deep analysis these could be estimated by the ethnographer to unpack $L$ but this would, of course, be a rather demanding and is an unlikely empirical procedure except perhaps when differing ethnographers reach inconsistent conclusions about $L$. Then some unpacking may reveal wherein differences lie. The ethnographer's estimate of the prior odds and likelihood ratio will of course depend upon the depth of the encounter with the informant and the 
estimate of his/her reliability, self-knowledge etc. Ethnographers may exhibit some reluctance to make estimates of this sort, but if they venture to draw conclusions about causal mechanism they may implicitly be doing so. If that is true, why not make it explicit for all to observe what they are doing?

\section{Meta-Ethnography}

The above outlined analysis may generally surrender multiple ethnographic estimates of the posterior odds for a given causal connection (i.e. odds $(H: \neg H \mid$ available evidence)). In a narrative context there may be estimates for each such causal connection (i.e. the action nodes in the associated andDAG). How should the various estimates, for a given causal connection, be aggregated into an overall estimation of the odds of the link?

The natural extension of the analysis, so far, is to adopt a Supra-Bayesian method (Clemens and Winkler, 1999) whereby a meta-ethnographer treats all of the posterior odds of each of the primary ethnographers as providing ethnographic evidence, alongside any prior she herself might entertain, then makes an estimate of the likelihood ratios, and thence calculates her posterior odds. This will of course involve all the unwieldy complications encountered above in estimating both the independent or dependent likelihood ratios. It, therefore, seems an unlikely aggregation candidate.

A linear pooling of the odds ratios of all ethnographers with equal weightings which sum to one is probably more promising in this respect and where there is no reason to elevate one ethnographer above another. This then amounts to simple averaging of the posterior odds ratios across all the primary ethnographers. An alternative is a normalised geometric pooling also with exponent weights when, once again, no primary ethnographer is given priority over any others.

Ethnographers are scrupulously careful in comparing and contrasting (i.e. aggregating and separating) subjective reports in order to arrive at an estimation of "what is going on". There is, however, as far as we can see, no available framework within which this procedure can be systematised. However, as we noted above, the Bayesian approach enables a common language of 
odds whereby comparisons may be made. Theories of probabilistic or odds pooling usually require that any aggregation technique should surrender unanimity (i.e. if all agree then this becomes the aggregate value), event wise independence (i.e. the aggregate only depends on the individual values) and Bayesian externality (i.e. it does not matter whether odds are updated before or after aggregation). Should these properties be taken as guides, on the meta-ethnographer's behalf, for causal inference where the basic evidence is subjective? Linear aggregation is unanimity preserving and event wise independent though fails to be Externally Bayesian. Geometric aggregation, on the other hand, is externally Bayesian and unanimity preserving, but not event-wise independent (Dietrich and Spiekermann, 2013).

We might start with a situation where all the primary ethnographers are in possession of the same set of statements $\left\{s_{i 1}, s_{i 2}, \ldots, s_{i m}\right\}$. This could be achieved either as a natural consequence of their research or by what is sometimes called behavioural aggregation where the primary ethnographers are brought together as a group where they share the statements they have elicited in interaction with various informants . They will of course not necessarily attribute the same credibility to identical statements, nor estimated Posterior Odds of the causal link. However, behavioural aggregation usually searches out unanimity of the aggregate estimate then, if achieved, this would seem to provide the strongest grounds for inferring a causal link. Failure to achieve unanimity using behavioural aggregation might, however be taken to invite either additional linear or geometrical aggregation. It is difficult, at this stage, to advocate any particular aggregation technique - the issue warrants further research, if the whole Bayesian framework towards causality is to be taken seriously.

\section{The Illustrative Empirical Example - A Further Look}

Returning now to the illustrative empirical example introduced above. Both subjective causal and counterfactual statements $\left(s_{i}\right)$ were solicited from five members of the governing body (including the one examined above) of the producer cooperative in respect of the collective action in appointing an 
independent manager. One of the five was the chairman of the governing body (informant 1) and another was the ex-chairman (informant 2). The first author of this paper continues in the role of ethnographer. With some prompting, the informants agreed upon the causally connected sets $X_{c}$ and $Y .{ }^{16}$ The ethnographer assumed that the prior odds of the credibility of the five conjoined statements of subjective and counterfactual causality was 1:1 (see footnote 13) and estimated the likelihood ratios of the credibility of this evidence attributable to each informant. With the assumption of the prior odds at 1:1 the likelihood ratios are then identical to the posterior odds of the credibility of the evidence. Table 1 gives the credibility beliefs afforded by the ethnographer to each informant. In this case the credibility of each informant is separately estimated rather than the overall credibility (equation 6).

[Table 1 about here]

The derived likelihoods of the causal hypothesis that

$$
X_{c} \rightarrow \text { governing body } D X_{o} \rightarrow Y
$$

attributable each of the five informants by the ethnographer are depicted in Table 2.

[Table 2 about here]

The credible evidence thus surrenders the odds that the causal link under investigation is correctly inferred at an average 6.6: 1 across informants. Inspection of Table 1 and 2 enables any audience of the research to appreciate how this overall support for the causal link is constituted (i.e. socially constructed) by the ethnographer and the informants. Recall that these calculations can also be placed alongside the text of the subjective statements. ${ }^{17}$

\footnotetext{
${ }^{16}$ In general acquiring agreement on the contents of set $X_{c}$ and Error! Main Document Only.Error! Main Document Only.Error! Main Document Only. $Y$ across all chosen informants is unlikely. The techniques outlined may then be used to test alternative hypotheses. This falls beyond the scope of this exploratory paper.

${ }^{17}$ The causal and counterfactual statements are so similar across informants that they are not reported here.
} 


\section{Conclusion}

We fully realise that many ethnographers will not find the contents of this paper at all congenial. Placing subjective evidence about causality within a Bayesian framework runs counter to many of their precepts about remaining entirely faithful to the actors own account of what they are doing. However, if small $N$ studies are to aspire to reveal causal connections then a systematic framework enabling comparison across informants and perhaps ethnographers must be found. Currently these procedures remain difficult to extract from reported studies (Abend et al, 2013). The techniques we have outlined here achieve precisely this, through the use of Bayesian inference. Only in depth applied work will show whether these sort of inference are intellectually revealing.

\section{References}

Abell, P. (1987). The Syntax of Social Life. The Theory and Method of Comparative Narratives. Oxford, Oxford University Press.

Abell, P., (1990). Supporting industrial cooperatives in developing countries: some Tanzanian experiences. Economic and Industrial Democracy, 11(4), pp.483-504.

Abell, P. (2009a). "History, Case Studies, Statistics and Causal Inference”. European Sociological Review, 25, 561-569.

Abell, P. (2009b). “A Case for Cases, Comparative Narratives in Sociological Explanation”, Sociological Methods and Research, 32, 1-33.

Abend, G. C. Petrie and M. Sauder (2013). "Styles of Causal Thought", American Journal of Sociology, 119(3), 602-654.

Bennett, A. and J. Checkel (eds.) (2015). Process Tracing in the Social Sciences; From Metaphor to Analytic Tool. Cambridge, Cambridge University Press.

Cardano (2009) Ethnography and Reflexivity. Notes on the Construction of Objectivity in Ethnographic Research. ELETTRONICO, - 1(2009), pp. 1-21., available at http://hdl.handle.net/2318/75133 
Cartwright, N. (2017), "Single Case Causes: What is Evidence and Why", in chao,H.K. and Reiss, J (eds), Philosophy of Science I Practice. Synthese library (Studies in Epistemology, logic Methodology and Philosophy of Science), Vol 379, Springer.

Chandra, U. (2014) Rashomon revisited: contending narratives on a gang rape in West Bengal Economic \& Political Weekly, Vol 49, Issue No. 17.

Clemen, R.T. and R.L. Winkler (1999). "Combining Probability Distributions from Experts in Risk Analysis". Risk Analysis, 19, 187-203.

Dietrich, F. and K. Spiekermann (2013). "Independent Opinions? On the Causal Foundations of Belief Formation and Jury Theorems". Mind, 122 (487)655-685.

Elman, C. Gerring, J. and J. Mahoney (2016). “Case Study Research: Putting Quant. into Qual.” Sociological Methods and Research, (3), 375-391.

Goldthorpe, J.H. (2001).“Causation, Statistics and Sociology”. European Sociological Review, 17, 120.

Hedström, P. (2005). Dissecting the Social: On the Principals of Analytical Sociology Cambridge, Cambridge University Press.

Heider, K G (1988): “The Rashomon Effect: When Ethnographers Disagree”, American Anthropologist, 90 (1): 73-81.

Hitchcock, C. (2007) “Prevention, Preemption, and the Principle of Sufficient Reason”, Philosophical Review, 116(4): 495-532.

Howson,C. and P. Urbach (2006). Scientific Reasoning: The Bayesian Approach. Caris Publishing. Mackie,J. L. (1980). The Cement of the Universe, Oxford, Oxford University Press.

Maher, P. (1993). Betting on Theories. Cambridge, Cambridge University Press.

Mahoney, J. (2000). "Strategies of Causal Inference in Small-N Analysis", Sociological Method and Research, 29(4), 387-424.

Mahoney J. (2012).“The logic of Process Tracing Tests in the Social Sciences.” Sociological Methods and Research, 45(4), 566-590. 
Mahoney, J. Goertz, G. and C.C. Ragin (2013). "Causal Models and Counterfactuals" in Stephen, L. Morgan, ed., Handbook of Causal Analysis for Social Research. New York, Springer.

Morgan, S. L. and C. Winship (2015). Counterfactuals and Causal Inference: Methods and Principles for Social Research ( $2^{\text {nd }}$ edition). Cambridge, Cambridge University Press.

Pearl, J. (2009). Causality Models, Reasoning and Inference. Cambridge, Cambridge University Press.

Ragin, C. C. (1987). The Comparative Method: Moving Beyond Qualitative and Quantitative Strategies. San Fransisco, University California Press.

Rohlfing I. (2013). Case Studies and Causal Inference. London, Palgrave, Macmillian.

Rubin, D. B. (2005). “Causal Inference Using Potential Outcomes; Design, Modelling, Decisions”. Journal of the American Statistical Association, 100, 322-31.

Schum, D. A. (1994). The Evidential Foundations of Probabilistic Reasoning. Evanston, North Weston University Press.

Small, M.L. (2013). “Causal Thinking and Ethnographic Research”. American Journal of Sociology, 19(3), 597-604.

Von Wright, G.H. (2004). Explanation and Understanding. Cornell, Cornell University Press.

Williamson, J. (2005). Bayesian Nets and Causality; Philosophical and Computational Foundations. Oxford, Oxford University Press. 
Ethnographic Causality

Table 1: Estimates of the credibility of statements by informants

\begin{tabular}{ccccccc}
\hline & $\boldsymbol{i}=\mathbf{1}$ & $\boldsymbol{i}=\mathbf{2}$ & $\boldsymbol{i}=\mathbf{3}$ & $\boldsymbol{i}=\mathbf{4}$ & $\boldsymbol{i}=\mathbf{5}$ & \\
\hline$L_{s_{i}}$ & $10: 1$ & $10: 1$ & $5: 1$ & $8: 1$ & $4: 1$ & $\overline{L_{s_{l}}}=6.6: 1$ \\
$\log L_{s_{i}}$ & 2.30 & 2.30 & 1.61 & 2.08 & 1.39 & $\sum_{i=1}^{m} \log \left(L_{s_{i}}\right)=9.68$ \\
\hline
\end{tabular}

Table 2: Justified belief in a Causal Relation

$$
i=1 \quad i=2 \quad i=3 \quad i=4 \quad i=5 \quad \text { Average }
$$

$\operatorname{Odds}\left(\boldsymbol{H}: \neg \boldsymbol{H} \mid \boldsymbol{s}_{i 1}, \boldsymbol{s}_{i 2}, \ldots, \boldsymbol{s}_{i 5}\right) \quad 10: 1 \quad 10: 1 \quad 5: 1 \quad 8: 1 \quad 4: 1 \quad 6.6: 1$

\begin{tabular}{|c|c|}
\hline $\log \left(\operatorname{Odds}\left(\boldsymbol{H}: \neg \boldsymbol{H} \mid \boldsymbol{s}_{i 1}, \boldsymbol{s}_{i 2}, \ldots, \boldsymbol{s}_{i 5}\right)\right)$ & 2.30 \\
\hline
\end{tabular}

